I was pleased to read Dr Crowley's in-depth understanding of the complexity and value of the altered state of consciousness. Thankfully, there are clinicians such as Dr Crowley with the confidence not to dismiss the non-ordinary state of consciousness as mere 'acute confusion', but to believe that psychedelics, and non-drug non-ordinary states of consciousness, can inform and enlighten us with new approaches to understanding the mechanisms (and associated pathologies) of the brain. Since the earliest human societies we have sought knowledge and healing from these states - perhaps now this technique can be utilised in a scientific and evidence-based approach to relieve the burden of anxiety disorders for today's patients.

I am most grateful to Dr Sandison for his kind and supportive words - and thank him for the correction regarding the date of the American Psychiatric Association conference in 1955. I share his astonishment at the medical profession's inability or unwillingness to embrace the therapeutic potential of psychedelic substances. This shortcoming is augmented by the fact that the hiatus in research over the past 40 years appears to have been for socio-political rather than scientific reasons - and it is those pioneering psychiatrists like $\mathrm{Dr}$ Sandison who are right to feel disheartened.

I am enthusiastic, however, at the current re-emergence of interest in this field. There are increasing numbers of randomised controlled trials of psychedelics (largely from the USA) and these may yield results that guide future therapeutic applications (http://www.maps.org; http:// www.heffter.org). There is also increasing interest in using psychedelics in consciousness research in the UK (http://www. beckleyfoundation.org).

I do hope that my article, and a forthcoming meeting to be held at the College (contact me at drbensessa@hotmail.com for further details), can help raise awareness of this subject. I also agree with $\mathrm{Dr}$ Sandison in his plea for continued support from the College to bring this subject to the attention of doctors in the UK.

Grof, S. (1990) The Holotropic Mind. New York: Harper Collins.

Masters, R. E. L. \& Houston, J. (1973) The Varieties of Psychedelic Experience (2nd edn). London: Turnstone Books.

B. Sessa The Park Hospital, Old Road, Headington, Oxford OX3 7LQ, UK. E-mail: drbensessa@hotmail.com

\section{Kraepelinian dichotomy}

Craddock \& Owen (2005) attribute the proposed demise of the Kraepelinian dichotomy to advances in genetic epidemiology, and rightly emphasise the need to integrate data across multiple domains in large numbers of people. However, it may also be important to use a population-based approach. This involves extra effort but avoids being misled by convenience samples which may not be representative of the population. This is illustrated by Fig. 1 in the editorial of Craddock \& Owen which suggests that prototypical schizophrenia and prototypical bipolar disorder are relatively rare in clinical populations. Work in population-based samples suggests that there is an early, insidious-onset psychosis with a poor outcome affecting predominantly men - a 'neurodevelopmental' form of schizophrenia which is very close to dementia praecox (Castle et al, 1998). This prototypical form of schizophrenia together with protoypical bipolar disorder accounts for $50 \%$ of people with psychosis in a treated prevalence sample, demonstrating the utility of Kraepelin's division. In our experience affective and non-affective psychoses can be accounted for by these prototypical forms and a further two latent classes which appear to be valid (Murray et al, 2005). Whether such empirically derived classes might provide better phenotypes for genetic studies is as yet undetermined.

Until biological markers are identified there is perhaps only one way to improve our classification. Large-scale, empirical, population-based studies of psychiatric symptoms, demography, course, treatment response and outcomes are suggested to reclassify these disorders from first principles and provide an atheoretical framework which may capture underlying pathophysiological substrates. Such studies should, as described by Craddock \& Owen, integrate both dimensional and categorical approaches but also require a developmental perspective across the life span. The debate about the Kraepelinian dichotomy illustrates the lack of evidence-based diagnostic classification in psychiatry as a discipline. It would be fitting if psychiatric genetics, which has been severely impeded by the lack of a robust nosology, focused the collective will of practitioners to establish the evidence base required for a psychiatric classification which at last reflects nature.
Castle, D. J., Wessely, S., van Os, J., et al (1998) Subtypes of schizophrenia. In Psychosis in the Inner City: The Camberwell First Episode Study, pp. 37-49. Hove: Psychology Press.

Craddock, N. \& Owen, M. J. (2005) The beginning of the end of the Kraepelinian dichotomy. British journal of Psychiatry, 186, 364-366.

Murray, V., McKee, I., Miller, P. M., et al (2005)

Dimensions and classes of psychosis in a population cohort: a four class, four dimension model of schizophrenia and affective psychoses. Psychological Medicine, 35, 499-510.

V. Murray Scottish Centre for Autism, Royal Hospital for Sick Children, Glasgow G3 8SJ, UK. E-mail: Val.Murray@yorkhill.scot.nhs.uk

Authors' reply: We are in full agreement with Dr Murray regarding the utility of large-scale, population-based studies. These are highly desirable and will, we hope, be facilitated by the recent establishment of the Mental Health Research Network (http://www.mhrn.info) under the auspices of the UK Clinical Research Collaboration (http://www.ukcrc.org). We also agree that longitudinal variables such as course, outcome and treatment response might be key to classification, as Kraepelin supposed. However, although we have not undertaken relevant population studies ourselves, we are not convinced that Kraepelinian dichotomous categories are any more useful in population-based samples than in clinical samples. We find the studies of Van Os and colleagues (e.g. Krabbendam et al, 2004) persuasive that dimensional measures are useful in describing psychosis-related morbidity in the general population and, contrary to the proposition of Dr Murray, we would expect dimensions to be more useful than categories in populations unselected for severe illness.

Finally, we would like to restate and further emphasise our optimism about the likely rate of progress in identifying biological markers that can validate psychiatric diagnoses. Markers (in the form of genetic polymorphisms) have already been identified that challenge current nosology. For example, using the Bipolar Affective Disorder Dimension Scale (which rates affective and psychotic dimensions; Craddock et al, 2004) in a study of over 600 cases each of schizophrenia and bipolar disorder, we have demonstrated that a risk variant within the Neuregulin 1 gene, which has been associated with risk of schizophrenia in several samples (reviewed in Craddock et al, 2005), may confer 
specific risk for a form of psychotic illness characterised by features of both mania and mood-incongruent psychosis (Green et al, 2005). Other findings of a similar nature are currently emerging from our own studies and those of other groups, and we anticipate that we are entering a period during which psychiatric research and practice will be placed on much firmer nosological foundations than has been possible in the past.

\section{Declaration of interest}

N.C. and M.J.O. are consultants to GlaxoSmithKline and have received grant funding and honoraria from GlaxoSmithKline, AstraZeneca and Lilly.

Craddock, N., Jones, I., Kirov, G., et al (2004) The Bipolar Affective Disorder Dimension Scale (BADDS) - a dimensional scale for rating lifetime psychopathology in bipolar spectrum disorder. BMC Psychiatry, 4, 19.

Craddock, N., O'Donovan, M. C. \& Owen, M. J. (2005) The genetics of schizophrenia and bipolar disorder: dissecting psychosis. Journal of Medical Genetics, 42, 288-299.

Green, F. K., Raybould, R., Macgregor, S., et al (2005) Operation of the schizophrenia susceptibility gene, neuregulin I, across traditional diagnostic boundaries to increase risk for bipolar disorder. Archives of General Psychiatry, 62, 642-648.

Krabbendam, L., Myin-Germeys, I., De Graaf, R. et al (2004) Dimensions of depression, mania and psychosis in the general population. Psychological Medicine, 34, 1177-1186.

N. Craddock, M. J. Owen Department of

Psychological Medicine, Henry Wellcome Building, School of Medicine, Cardiff University, Heath Park, Cardiff CFI4 4XN, UK.

E-mail: craddockn@Cardiff.ac.uk

\section{CBT for refractory symptoms in schizophrenia}

Valmaggia et al (2005) report an interesting randomised controlled trial evaluating cognitive-behavioural therapy (CBT) for refractory psychotic symptoms of schizophrenia resistant to atypical antipsychotic medication. They conclude that patients should not be excluded from psychological help on the grounds that they are too ill to benefit from therapy, and CBT for psychotic symptoms should be available in in-patient facilities.

We feel the conclusions drawn by the authors do not truly reflect their results. Valmaggia et al report that their primary hypothesis was that CBT would be more effective than supportive counselling in reducing auditory hallucinations and delusional beliefs. They used the Positive and Negative Syndrome Scale (PANSS) and Psychotic Symptoms Rating Scale (PSYRATS) to measure outcomes. The post-treatment score on the PANSS positive sub-scale of those receiving CBT was not significantly different from that of the control group. On the PSYRATS no significant effect was found on the delusions. Benefits of CBT were found on the auditory hallucinations scale for physical characteristics and cognitive interpretation but not for emotional characteristics. However, the benefits noticed were not sustained at follow-up. It would have been helpful if the authors had used an a priori definition of what constitutes a clinically meaningful improvement and provided the actual figures for the dichotomous outcome.

Also, if we look at the numbers needed to treat (NNT) calculations, the authors have accurately reported the lack of statistical significance (PANSS positive symptom scale, $\mathrm{NNT}=8,95 \%$ CI $3-\infty$; PSYRATS factor $2, \mathrm{NNT}=6,95 \%$ CI $2-\infty$; delusion scale factor $1, \mathrm{NNT}=4,95 \%$ CI $2-\infty$; factor $2, \mathrm{NNT}=12,95 \%$ CI $3-\infty)$. The only finding with reasonable confidence intervals seems to be cognitive interpretation on the auditory hallucination scale of the PSYRATS (NNT $=3$, 95\% CI 2-13). The authors also draw our attention to the fact that clozapine is effective in $32 \%$ of cases in producing a clinical improvement $(\mathrm{NNT}=5,95 \%$ CI 4-7; Wahlbeck et al, 1999). They seem to suggest that the figures from the current study reveal the effects of CBT to be similar to clozapine. However, it should be noted that this figure reported by Wahlbeck et al is for global improvement, whereas Valmaggia et al do not give any figures for global improvement and hence in our opinion these results are not comparable. To conclude from these results that CBT could induce a change in psychotic symptoms seems to be overestimating the beneficial effects.

Patients with schizophrenia who are resistant to clozapine form one of the most difficult-to-treat groups. Jones et al (2004) concluded that trial-based data supporting the wide use of CBT for people with schizophrenia or other psychotic illnesses are far from conclusive. The randomised controlled study of Valmaggia et al evaluating interventions in this population is welcome.

Jones, C., Cormac, I., Silveira da Mota Neto, J. I., et al (2004) Cognitive behaviour therapy for schizophrenia. Cochrane Database of Systematic Reviews, issue 4. Oxford: Update Software.

Valmaggia, L. R., Van der Gaag, M., Tarrier, N., et al (2005) Cognitive-behavioural therapy for refractory psychotic symptoms of schizophrenia resistant to atypical antipsychotic medication: Randomised controlled trial. British Journal of Psychiatry, 186 324-330.

Wahlbeck, K., Cheine, M. \& Essali, M. A. (1999) Clozapine versus typical neuroleptic medication for schizophrenia. Cochrane Database of Systematic Reviews, issue 4. Oxford: Update Software.

M. Jayaram, P. Hosalli St Mary's House, St Mary's Road, Leeds LS7 3JX, UK.

E-mail: Mahesh.Jayaram@leedsmh.nhs.uk

\section{Olanzapine co-therapy in bipolar disorder}

Baker et al (2004) report an interesting post hoc analysis from a randomised doubleblind, placebo-controlled study evaluating the efficacy of olanzapine co-therapy in patients with bipolar disorder who had adequate responses to valproate or lithium monotherapy (Tohen et al, 2002). The authors describe a secondary analysis assessing response among dysphoric and non-dysphoric patients with bipolar I disorder.

The authors conclude that olanzapine in combination with either lithium or valproate was effective in improving the severity of depressive symptoms coexisting with acute mania. This conclusion is based on statistically significant differences in mean changes in Hamilton Rating Scale for Depression (HRSD) score. However, the authors have not reported the standard deviations for these mean changes. Hence it is difficult to ascertain whether the data are skewed. It is possible that a few patients showing large changes on the HRSD could have skewed the data. It was also puzzling that the authors reported that the difference in the HRSD score between combination and monotherapy groups was larger for dysphoric patients. One would expect participants in the non-dysphoric group to have much lower baseline scores so that there would be less chance of a significant reduction. (The mean HRSD baseline score in the non-dysphoric group was 10.42 (s.d.=5.27) and in the dysphoric group 25.18 (s.d.=4.62).

We are also of the view that reporting study outcomes in terms of mean changes on a rating scale does not provide meaningful information for clinicians. Reporting results using dichotomous outcome 